\title{
A new method for rapid comparison of protein binding pockets by capturing spatial distributions
}

\author{
Timo Krotzky ${ }^{*}$, Gerhard Klebe \\ From 9th German Conference on Chemoinformatics \\ Fulda, Germany. 10-12 November 2013
}

Efficient determination of structural similarities between protein binding pockets is an important challenge in computational chemistry. A degree of similarity in the mutual comparison is often estimated in terms of graphs and by calculating a metric such as the maximum shared common subgraph. Cavbase [1] was developed as a tool for the automatic detection, storage and classification of putative protein binding sites. Cavbase assigns so-called pseudocenters to the cavity-flanking amino acids, which characterize their physicochemical properties with respect to molecular recognition. Subsequently, the pseudocenters are used as graph nodes to accomplish mutual binding site comparisons. This way of modeling protein binding sites, however, tends to be computationally very demanding, which often leads to very lengthy evaluations of the similarity measures.

In this study we propose Rapid Pocket Matching using Distances (RAPMAD), a new modeling formalism for Cavbase entries which allows for highly efficient similarity calculations. Here, protein binding sites are represented by sets of distance histograms based on specific spatial reference points [2] in order to characterize the distribution of pseudocenters within the cavity. The histograms can be both generated and compared with linear complexity. Attaining a speed of approximately 20,000 comparisons per second, pocket comparisons across large datasets and even screenings of entire databases become easily feasible.

We demonstrate the discriminative power and the orders of magnitude faster runtime of this novel method by carrying out several classification and retrieval experiments. Among others, datasets of protein cavities hosting specific cofactors are used for classification experiments, where RAPMAD results in a considerably higher rate of correct classifications compared to other

\footnotetext{
*Correspondence: krotzky@uni-marburg.de

Department of Pharmaceutical Chemistry, Philipps-Universität, Marburg,
} 35032, Germany

alternative approaches while it requires only a fraction of their runtime. Moreover, a set of proteases [3] was investigated, where it turned out that RAPMAD is able to distinguish between different Merops clans such as serine or metallo proteases.

Published: 11 March 2014

\section{References}

1. Schmitt $S$, Kuhn D, Klebe $G$ : A new method to detect related function among proteins independent of sequence and fold homology. $\mathrm{J} \mathrm{Mol} \mathrm{Biol}$ 2002, 323(2):387-406.

2. Ballester PJ, Richards WG: Ultrafast shape recognition to search compound databases for similar molecular shapes. J Comput Chem 2007, 28(10):1711-1723.

3. Glinca S, Klebe G: Cavities Tell More than Sequences: Exploring Functional Relationships of Proteases via Binding Pockets. J Chem Inf Model 2013, 53(8):2082-2092.

Cite this article as: Krotzky and Klebe: A new method for rapid comparison of protein binding pockets by capturing spatial distributions. Journal of Cheminformatics 2014 6(Suppl 1):P45.
- available free of charge to the entire scientific community

- peer reviewed and published immediately upon acceptance

- cited in PubMed and archived on PubMed Central

- yours - you keep the copyright

Submit your manuscript here:

http://www.chemistrycentral.com/manuscript/ 